Louisiana State University

LSU Digital Commons

$12-1-2010$

\title{
Dynamical symmetries in contemporary nuclear structure applications
}

A. I. Georgieva

Institute for Nuclear Research and Nuclear Energy Bulgarian Academy of Sciences

M. I. Ivanov

Institute for Nuclear Research and Nuclear Energy Bulgarian Academy of Sciences

S. L. Drenska

Institute for Nuclear Research and Nuclear Energy Bulgarian Academy of Sciences

K. D. Sviratcheva

Louisiana State University

J. P. Draayer

Louisiana State University

Follow this and additional works at: https://digitalcommons.Isu.edu/physics_astronomy_pubs

\section{Recommended Citation}

Georgieva, A., Ivanov, M., Drenska, S., Sviratcheva, K., \& Draayer, J. (2010). Dynamical symmetries in contemporary nuclear structure applications. Physics of Particles and Nuclei, 41 (7), 1105-1107.

https://doi.org/10.1134/S1063779610070270

This Article is brought to you for free and open access by the Department of Physics \& Astronomy at LSU Digital Commons. It has been accepted for inclusion in Faculty Publications by an authorized administrator of LSU Digital Commons. For more information, please contact ir@lsu.edu. 


\title{
DYNAMICAL SYMMETRIES IN CONTEMPORARY NUCLEAR STRUCTURE APPLICATIONS
}

\author{
A. I. Georgieva ${ }^{1}$, M. I. Ivanov ${ }^{1}$, S. L. Drenska ${ }^{1}$, \\ K.D. Sviratcheva ${ }^{2}$, J.P. Draayer ${ }^{2}$ \\ ${ }^{1}$ Institute for Nuclear Research and Nuclear Energy, Bulgarian Academy of Sciences, Sofia \\ ${ }^{2}$ Louisiana State University, Baton Rouge, USA
}

In terms of group theory - the language of symmetries, the concept of spontaneous symmetry breaking is represented in terms of chains of group-subgroup structures that define the dynamical symmetry of the system under consideration. This framework enables exact analytic solutions of the associated eigenvalue problems.

We review two types of applications of dynamical symmetries in contemporary theoretical nuclear structure physics: first for a classification of the many-body systems under consideration, with respect to an important characteristic of their behavior; and second for the creation of exactly solvable algebraic models that describe specific aspects of this behavior. This is illustrated with the boson and fermion realizations of symplectic structures; in the first case with an application of the $s p(4, R)$ classification scheme of even-even nuclei within the major nuclear shells and next with an application of the $s p(4)$ microscopic model for the description of isovector pairing correlations.

PACS: 21.60.-n

\section{INTRODUCTION}

The nucleus is a complex many-body structure that exhibits various singleparticle and collective modes of behavior of its constituents, the protons and neutrons. An important and successful approach in its investigation is the study of the symmetries of the system, in particular in their manifestation in its collective behavior. A major brake through in this respect is the notion of spontaneous breaking of symmetries, introduced by N. N. Bogolyubov in the statistical physics. On the mathematical language of symmetries, which is the group theory, the notion of spontaneous breaking of symmetries [1] is introduced by chains of groupsubgroup structures. These chains are also named dynamical symmetries [2]. The dynamical symmetries represent specific cases, in which:

- First of all, one can classify the basis states of the system under consideration by means of the eigenvalues of the reduction operators. This approach is used and illustrated below for the creation of the $S p(4, R)$ classification scheme [3] of the even-even nuclei within major nuclear shells. We present one of its successful 
applications, for a unified description of the yrast energies of almost all eveneven nuclei [4], that demonstrate regular behavior when empirically investigated within their classification.

- Next, employing dynamical symmetries, we obtain exact analytic solutions of the eigenvalue problems for the physical observables under consideration. One such an example is the microscopic model [5] based on the $S p(4)$ symmetry, which was used to describe isovector pairing correlations in nuclei.

\section{THE $S p(4, R)$ CLASSIFICATION SCHEME AND ITS APPLICATIONS}

We outline briefly the algebraic approach, which is the basis for the classification scheme used in [4]. It is rather similar to the methods of classifying the elementary particles. The classification procedure can be understood by introducing a classification group contained in a bigger dynamical group, which spans the considered nuclear characteristics of all the classified nuclei. The reduction of the boson representation of the classification group $S p(4, R)$ to its compact $u(2)$ and noncompact $u(1,1)$ subalgebras [6]

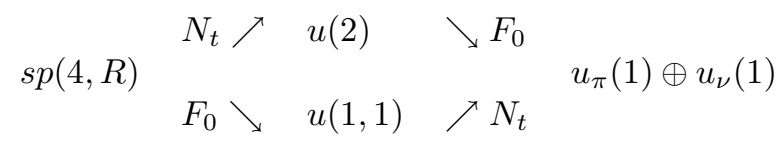

is the mathematical basis behind this scheme. As illustrated in (1) the reduction is realized by means of the operator of the total number of valence particles $N_{t}=\left(N_{\pi}+N_{\nu}\right)$, which is the first-order invariant of $u(2)$, and the operator of the third projection of the $F$-spin $-F_{0}=(1 / 2)\left(N_{\pi}-N_{\nu}\right)$, which does not differ essentially from the first-order Casimir for $u(1,1) . N_{t}$ reduces the space $H$, in which acts the boson representation of $\operatorname{sp}(4, R)$, into a direct sum of a totally symmetric irreducible unitary representations (IUR) of $s u(2)$, labeled by $N_{t}=0,2,4, \ldots$ (even $H_{+}$) or $N_{t}=1,2,3, \ldots$ (odd $H_{-}$). The operator $F_{0}$ reduces the space $H$ to the ladder series of $u(1,1)$, defined by its fixed eigenvalues. The same operator $F_{0}$ reduces each $u(2)$ representation (fixed value of $N_{t}$ ) to the representations of $u_{\pi}(1) \oplus u_{\nu}(1)$ labeled by $N_{\pi}$ and $N_{\nu}$, respectively. The same is obtained by reducing the $u(1,1)$ ladders with the operator $N_{t}$.

The relation of the algebraic operators used in the classification scheme to the nuclear characteristics in the valence shell, is rather natural, when $N_{\pi}=$ $(1 / 2)\left(N_{p}-Z^{(1)}\right)$ and $N_{\nu}=(1 / 2)\left(N_{n}-N^{(1)}\right)$ are interpreted as the number of proton and neutron valence pairs of the nucleus from a given shell, in which $Z^{(1)}$ and $N^{(1)}$ are the numbers of protons and neutrons of the double magic nucleus at the beginning of the shell. Then $N_{t}$ and $F_{0}$ are exactly the operators reducing the $s p(4, R)$ spaces, and their interpretation corresponds to the one of the IBM II [7], as the total number of valence bosons and the third projection of the $F$-spin. 
In [4] we were able to show that the low-lying yrast energies exhibit a smooth and periodic behavior in each $F_{0}$ multiplet, which allows their unified description in terms of the classification quantum numbers $N_{t}$ and $F_{0}$ of the $S p(4, R)$ classification scheme. On this basis the following generalized phenomenological formula for the energies of the ground-state band was obtained [4]:

$$
E_{\text {yrast }}\left(h_{k}, I, \omega\right)=\alpha\left\{h_{k}\right\} L(L+w) .
$$

The inertial parameter $\alpha\left\{h_{k}\right\}$ is a second-order polynomial of $h_{k} \equiv\left\{N_{t}, F_{0}\right\}$ for each of the nuclei $(k)$ in a fixed nuclear shell. The phenomenological parameters of the polynomial $\alpha\left\{h_{k}\right\}$ are determined in a fitting procedure overall for all nuclear shells [4].

The second term of Eq. (2) represents the generalized collective interaction characterized by the geometrical parameter $w$. The latter has the physical meaning of a measure for the interplay between the vibrational and the rotational collective modes (2) and so reflects the respective changes in nuclear shape. The starting values of $w$ have been determined [4] for each of the classified nuclei by using the experimental yrast energy ratios $R_{4}=\frac{E\left(4_{1}^{+}\right)}{E\left(2_{1}^{+}\right)}$and their theoretical relation to the $w$ parameter $R_{4}(w)=\frac{E\left(4_{1}^{+}\right)}{E\left(2_{1}^{+}\right)}=2+\frac{4}{2+w}$. Hence, the values of the parameter $w$, like $R_{4}(w)$, reflect the changes of the shapes in heavy nuclei from almost spherical at the beginning of the shell to axially deformed in the midshell region and back towards spherical at its closure. In general, its specific value for each of the classified nuclei is shown to provide a reliable criteria for the development of the nuclear collectivity.

In summary, the successful systematic [3] of the yrast energies in [4] is based on the introduction of $F$-spin multiplets, acting in the spaces of the boson representations of the noncompact $S p(4, R)$.

\section{THE APPLICATION OF THE $s p(4)$ DYNAMICAL SYMMETRY TO PAIRING CORRELATIONS IN NUCLEI}

Based on a fermion realization of $s p(4)$ (isomorphic to $s o(5)$ ) [8] our aim is to investigate the properties of the pairing interaction by considering the symplectic algebra to be a dynamical symmetry algebra. This yields an isospin breaking phenomenological Hamiltonian written in terms of the group generators. The limiting cases of $s p(4)$ correspond to different reductions to $u(2)$ and reveal the properties of different coupling modes of the isovector pairing interaction. A generalization to multiple shells provides a classification scheme for nuclear ground states when the valence nucleons occupy more than a single orbit. This 
introduces shell structure into the theory and allows for an investigation of the dependence of pairing correlations on the dimensionality of the model space.

The $s p(4)$ algebra is realized in terms of creation and annihilation fermion operators with the anticommutation relations $\left\{c_{j, m, \sigma}, c_{j^{\prime}, m^{\prime}, \sigma^{\prime}}^{\dagger}\right\}=\delta_{j, j^{\prime}} \delta_{m, m^{\prime}} \delta_{\sigma, \sigma^{\prime}}$, $\left\{c_{j, m, \sigma}^{\dagger}, c_{j^{\prime}, m^{\prime}, \sigma^{\prime}}^{\dagger}\right\}=0,\left\{c_{j, m, \sigma}, c_{j^{\prime}, m^{\prime}, \sigma^{\prime}}\right\}=0$, where these operators create (annihilate) a particle of type $\sigma= \pm 1 / 2$ (proton/neutron) in a state of total angular momentum $j$ (half-integer) with projection $m$. For $p$ orbits the dimension of the shell is $2 \Omega=\Sigma_{j}(2 j+1)$. In addition to the number operator $N=N_{1}+N_{-1}$ and the isospin projection $T_{0}=\left(N_{1}-N_{-1}\right) / 2$, the generators of $S p_{q}(4)$ are

$$
\begin{gathered}
T_{ \pm}=\frac{1}{\sqrt{2 \Omega}} \sum_{j m} c_{j m, \pm 1 / 2}^{\dagger} c_{j m, \mp 1 / 2}, \\
A_{\mu}^{\dagger}=\frac{1}{\sqrt{2 \Omega\left(1+\delta_{\sigma \sigma^{\prime}}\right)}} \sum_{j m}(-1)^{j-m} c_{j m, \sigma}^{\dagger} c_{j,-m, \sigma^{\prime}}^{\dagger}, \quad A_{\mu}=\left(A_{\mu}^{\dagger}\right)^{\dagger} .
\end{gathered}
$$

The first two are related to the valence isospin, and the rest, $A_{0, \pm 1}^{\dagger}\left(A_{0, \pm 1}\right)$, create (annihilate) a pair of total angular momentum $J^{\pi}=0^{+}$and isospin $T=1$.

The basis states are constructed as $(T=1)$-paired fermions

$$
\left.\mid n_{1}, n_{0}, n_{-1}\right)=\left(A_{1}^{\dagger}\right)^{n_{1}}\left(A_{0}^{\dagger}\right)^{n_{0}}\left(A_{-1}^{\dagger}\right)^{n_{-1}}|0\rangle
$$

where $n_{1}, n_{0}, n_{-1}$ are the total number of pairs of each kind, $p p, p n, n n$, respectively.

The model provides for a classification scheme of nuclei and their isovectorpaired $0^{+}$states (Table). The total number of the valence particles, $N$, enumerates the rows and the valence isospin projection, $T_{0}$, enumerates the columns. Isotopes (isotones) of an element are situated along the right (left) diagonals, and the rows consist of isobars for a given mass number.

\begin{tabular}{|c|c|c|c|c|c|}
\hline$N / T_{0}$ & 0 & -1 & -2 & -3 & -4 \\
\hline 0 & ${ }_{20}^{40} \mathrm{Ca}_{20}$ & & & & \\
\hline 2 & ${ }_{21}^{42} \mathrm{Sc}_{21}$ & ${ }_{20}^{42} \mathrm{Ca}_{22}$ & & & \\
\hline 4 & ${ }_{22}^{44} \mathrm{Ti}_{22}$ & ${ }_{21}^{44} \mathrm{Sc}_{23}$ & ${ }_{20}^{44} \mathrm{Ca}_{24}$ & & \\
\hline 6 & ${ }_{23}^{46} \mathrm{~V}_{23}$ & ${ }_{22}^{46} \mathrm{Ti}_{24}$ & ${ }_{21}^{46} \mathrm{Sc}_{25}$ & ${ }_{20}^{46} \mathrm{Ca}_{26}$ & \\
\hline 8 & ${ }_{24}^{48} \mathrm{Cr}_{24}$ & ${ }_{23}^{48} \mathrm{~V}_{25}$ & ${ }_{22}^{48} \mathrm{Ti}_{26}$ & ${ }_{21}^{48} \mathrm{Sc}_{27}$ & ${ }_{20}^{48} \mathrm{Ca}_{28}$ \\
\hline
\end{tabular}

Classification scheme of nuclei in the $1 f_{7 / 2}$ level with a ${ }^{40}$ Ca core $(\Omega=4)$. The shape of the table is symmetric with respect to the sign of $T_{0}$ and $N-2 \Omega$ 
The isospin breaking model Hamiltonian [5]

$$
\begin{array}{r}
H=-\epsilon N-G A_{0}^{\dagger} A_{0}-F\left(A_{+1}^{\dagger} A_{+1}+A_{-1}^{\dagger} A_{-1}\right)-\frac{E}{2 \Omega}\left(T^{2}-\frac{3 N}{4}\right)- \\
-C \frac{N(N-1)}{2}-\left(D-\frac{E}{2 \Omega}\right)\left(T_{0}^{2}-\frac{N}{4}\right)
\end{array}
$$

includes isovector $(T=1)$ pairing interaction, diagonal isoscalar $(T=0)$ force and a symmetry term. A fit to the experimental energies of the lowest isovectorpaired $0^{+}$state (the ground state for even-even and some $[N \approx Z]$ odd-odd nuclei and the isobaric analog excited state for the rest odd-odd nuclei) leads to a good theoretical prediction of the relevant energies [5]. The interaction strength parameters are estimated in a fit with a small uncertainty for three groups of nuclei $\left(1 d_{3 / 2}, 1 f_{7 / 2}, 1 f_{5 / 2} 2 p 1 g_{9 / 2}\right)$ [5]. The values for the pairing strengths are found to lie on a curve that decreases with mass number, $G / \Omega=(25.7 \pm 0.5) / A$ ( $R^{2}=0.99$, goodness-of-fit measure), $F / \Omega=(23.9 \pm 1.1) / A\left(R^{2}=0.96\right)$. This allows for their further prediction for the region of $1 g_{7 / 2} 2 d 3 s 1 h_{11 / 2}$, where the energy spectrum of $Z \approx N$ and proton-rich nuclei is not yet measured.

Among all the interactions in the model, only the pn isovector correlations show a prominent peak in energy around $Z=N$. The $p n$ pairing also plays a significant role in reproducing the experimental data for the $S_{2 p}$ two-proton separation energy around $Z=N$ [9]. The zero point of $S_{2 p}$ determines the two-proton-drip line, which according to the $S p(4)$ model lies near the following nuclei: $\mathrm{Ge}_{28}, \mathrm{Ga}_{29}, \mathrm{Se}_{30}, \mathrm{As}_{31}, \mathrm{Kr}_{32}, \mathrm{Br}_{33}, \mathrm{Sr}_{34}, \mathrm{Rb}_{35}, \mathrm{Zr}_{36}, \mathrm{Y}_{37}, \mathrm{Zr}_{38}, \mathrm{Y}_{39}$, $\mathrm{Mo}_{40}, \mathrm{Nb}_{41}, \mathrm{Ru}_{42}, \mathrm{Tc}_{43}, \mathrm{Pd}_{44}, \mathrm{Rh}_{45}, \mathrm{Cd}_{46}, \mathrm{Ag}_{47}$. Different types of discrete derivatives of the energy function [9] reveal additional features of the nuclear interaction and provide for a good test of the model.

\section{CONCLUSIONS}

In the presented investigations, based on the boson and fermion realizations of the symplectic $S p(4, R)$ and $S p(4)$ algebras and their reductions, we use the notion of dynamical symmetries in order to interpret and predict properties of the nuclear collective structure in comparison with experimental data.

We show that an application of the $S p(4, R)$ classification scheme to eveneven nuclei within major nuclear shells, focused on a systematic empirical investigation of their collective features, leads to a unified phenomenological description and explanation of the observed regularities in their collective behavior. The outcomes further suggest that the analysis could be used to make predictions about heretofore unknown results.

We develop a microscopic model with $S p(4)$ dynamical symmetry for a description of the global behavior as well as fine effects in the energies of the 
completely paired $0^{+}$states, generated by the isovector pairing correlations in even $32 \leqslant A \leqslant 100$ nuclei, for which the protons and neutrons occupy the same major shell.

The above investigations of the symplectic dynamical symmetries in nuclear structure illustrate the importance and influence of the concepts of spontaneous symmetry breaking, that was suggested by N. N. Bogolyubov, as applying generally in quantum mechanics, and in particular in the nuclear structure theory.

Acknowledgements. This work was partly supported by Grants Number $\Phi-1501$ and $D I D 02 / 16$ of the Bulgarian National Foundation for scientific research and a grant for scientific collaboration with the Bogoliubov Laboratory of Theoretical Physics of JINR, Dubna. We acknowledge also support by the U.S. National Science Foundation (PHY-0500291 \& OCI-0904874), and the Southeastern Universities Research Association (http:/www.sura.org).

\section{REFERENCES}

1. Cseh J. // Bulg. J. Phys. 2006. V.33, No.s1. P.910-917.

2. Dashen R.F., Gell-Mann M. // Phys. Lett. 1965. V.17. P. 142; Dothan Y., Gell-Mann M., Ne'eman Y. // Ibid. P. 145; Dothan Y., Gell-Mann M., Ne'eman Y. // Ibid. P. 148.

3. Georgieva A. et al. // Intern. J. Theor. Phys. 1989. V. 28. P. 769.

4. Drenska S. et al. // Phys. Rev. C. 1995. V.52. P.1853;

Drenska S., Georgieva A., Minkov N. // Phys. Rev. 2002. C. V.65. P. 054303.

5. Sviratcheva K. D., Georgieva A. I., Draayer J. P. // J. Phys. G: Nucl. Part. Phys. 2003. V.29. P. 1281;

Sviratcheva K.D., Georgieva A.I., Draayer J.P. // Phys. Rev. C. 2004. V. 70. P. 064302.

6. Georgieva A. et al. // Intern. J. Theor. Phys. 1985. V.25. P. 1181.

7. Arima A. et al. // Phys. Lett. B. 1977. V.66. P. 205; Elliott J. P. // Rep. Progr. Phys. 1985. V.48. P. 171.

8. Sviratcheva K. D. et al. // J. Phys. A: Math. Gen. 2001. V.34. P. 8365; Sviratcheva K. D., Georgieva A. I., Draayer J. P. // J. Phys. A: Math. Gen. 2003. V. 36. P. 7579.

9. Sviratcheva K.D., Georgieva A.I., Draayer J.P. // Phys. Rev. C. 2004. V.69. P. 024313. 